\title{
An effective rhizoinoculation restraints arsenic translocation in peanut and maize plants exposed to a realistic groundwater metalloid dose
}

\author{
J.M. Peralta ${ }^{1}$, C.N. Travaglia ${ }^{1}$, R.A. Gil ${ }^{2}$, A. Furlan ${ }^{1}$, S. Castro ${ }^{1}$ \& E.C. Bianucci ${ }^{1}$ \\ ${ }^{1}$ Departamento de Ciencias Naturales, Facultad de Ciencias Exactas, Físico-Químicas y Naturales, \\ Universidad Nacional de Río Cuarto, Córdoba, Argentina \\ ${ }^{2}$ Laboratorio de Espectrometría de Masas - Instituto de Química de San Luis (CCT-San Luis), \\ Área de Química Analítica, Universidad Nacional de San Luis, San Luis, Argentina
}

\begin{abstract}
Groundwater with high arsenic (As) concentration constitutes a serious problem for crops, since roots can accumulate the metalloid acting as the first stage of As distribution in the trophic chain. The aim of this research was to elucidate the impact of a realistic $\mathrm{As}(\mathrm{V})$ dose in peanut and maize plants and to determine the contribution of plant growth promoting bacteria (PGPB) to metalloid translocation in both crops. The results obtained revealed that rhizoinoculation of plants exposed to metalloid, contributed not only to improve growth but also to reduce As transport to shoots. Hence, inoculation of peanut and maize with the correct PGPB partner prevents metalloid translocation in plants avoiding possible fruit contamination.
\end{abstract}

\section{INTRODUCTION}

Arsenic (As) is a harmful metalloid that impacts on crops acting as the first stage of As distribution in the trophic chain. In Córdoba province (Argentina), 90\% of the region is affected by groundwater As concentrations that exceeds the maximum allowed level in drinking water (FAO, 2015), being arsenate (As(V)) the prevalent arsenic form (Blarasin et al., 2014). Peanut and maize plants constitute important crops in Córdoba, as they represent approximately $90 \%$ of the Argentinean production. These crops establish interactions with plant growth promoting bacteria (PGPB) improving yield (Glick, 1995). Given the As amounts determined in groundwater, the metalloid absorption by crop plants could represent an agricultural and human health problem. Therefore, our experiments were conducted to elucidate the impact of a realistic $\mathrm{As}(\mathrm{V})$ dose in peanut and maize plants growth and to determine the contribution of PGPB to metalloid translocation in both plants.

\section{METHOD/EXPERIMENTAL}

\subsection{Bacterial strains}

Bradyrhizobium sp. SEMIA6144 and Bradyrhizobium sp. C-145 strains were obtained from MIRCEN (Brazil) and INTA (Argentina), respectively. Azospirillum brasilense AZ39 and A. brasilense CD (ATTCC 29710) were provided by IMIZA-INTA (Argentina) and EMBRAPA (Brasil), respectively. Bradyrhizobial strains were cultivated in liquid YEM medium
(Vincent, 1970) and Azospirillum strains in liquid Nfb medium (Dobereiner, 1988).

\subsection{Plant material and experimental design}

Peanut and maize seeds were supplied by "El Carmen S.A" and DEKALB respectively. Seeds were surface sterilized (Vincent, 1970) and pre-germinated seeds were transferred to a Leonard Jar system containing sterile substrate sand:perlite (2:1) with Hoagland's nutrient solution (Hoagland and Arnon, 1950) devoid of $\mathrm{As}(\mathrm{V})$ (control) or containing $3 \mu \mathrm{M} \mathrm{As}(\mathrm{V})$, supplied as $\mathrm{Na}_{2} \mathrm{HAsO}_{4} .7 \mathrm{H}_{2} \mathrm{O}$ (the metalloid concentration found in groundwater of some areas of Córdoba). Plants were divided in two groups (non-inoculated and inoculated) and grown in a controlled environment for 30 days. At harvest, growth and nodulation variables and nitrogen content (by Kjeldahl method) were determined.

\subsection{Arsenic accumulation and translocation in plant tissues}

Metalloid concentration was determined in peanut and maize shoots, roots and nodules (legume) by using an inductively coupled plasma mass spectrometry (ICPMS) (Sobrino-Plata et al., 2009). The translocation factor (TF) was calculated as Singh and Agrawal (2007).

\subsection{Statistical analysis}

Experiments were conducted in a completely randomized design and repeated three times. The data were 
analyzed using ANOVA and Duncan's test at $\mathrm{P}>0.05$. Prior to the test of significance, normality and homogeneity of variance were verified using the modified Shapiro-Wilk and Levene tests, respectively.

\section{RESULTS AND DISCUSSION}

\subsection{Arsenic impact on peanut and maize growth}

Peanut plants exposed to $\mathrm{As}(\mathrm{V})$ showed a significant reduction in shoot dry weight only in non-inoculated plants, without differences in the root dry weight (Table 1). Nodule number and dry weight of peanut plants were reduced by As. Nevertheless, the comparison between bradyrhizobial strains revealed that Bradyrhizobium sp. C-145 had a better behavior than Bradyrhizobium sp. SEMIA6144 since these variables were significantly higher in both control and treated condition. In the control treatment, nitrogen content was similar in plants inoculated with either bradyrhizobial strain, and significantly greater than in non-inoculated plants. However, As(V) addition caused a reduction of nitrogen content only in plants inoculated with Bradyrhizobium sp. SEMIA6144. In maize plants, all growth variables as well as nitrogen content were decreased by metalloid addition irrespective of the inoculation condition tested. Remarkably, Azospirillum strains allowed greater bi-ological nitrogen fixation (BNF) in plants, as determined by nitrogen content, compared to non-inoculated plants even when the crops were exposed to the metalloid (Table 1). In support of our results a significant reduction of soybean, peanut, lupin and maize growth were observed when exposed to $\mathrm{As}(\mathrm{V})$, being the generation of reactive oxygen species the main reason of cellular toxicity (Bianucci et al., 2017, 2018; Finnegan \& Chen, 2012; Lu et al., 2017; Vázquez-Reina et al., 2005). In addition, reduction of nodulation variables was also observed in lupin and soybean plants exposed to As (Bianucci et al., 2018; Carpena et al., 2006). It is known that the interaction established between peanut or maize with PGPB, as used in this research, is important since it fulfils a plant's $\mathrm{N}$ demand via the BNF process (Glick, 1995). However, reports evaluating the impact of As in the symbiotic interaction between peanut and rhizobia is scarce, even more in the maize-PGPB interaction. According to our results, it is possible to suggest that the inoculation of peanut with bradyrhizobia or maize with Azospirillum contribute to enhance growth variables in crops exposed to a realistic As groundwater dose. In this sense, Bradyrhizobium sp. C-145 and A. brasilense Az39 strains had the better behavior among tested strains.

\subsection{Arsenic accumulation and translocation in peanut and maize plants}

Arsenic accumulation by peanut plants was mainly detected in roots, followed by nodules and finally in shoots, regardless of the inoculation condition (Table 2). Metalloid content in shoots of plants

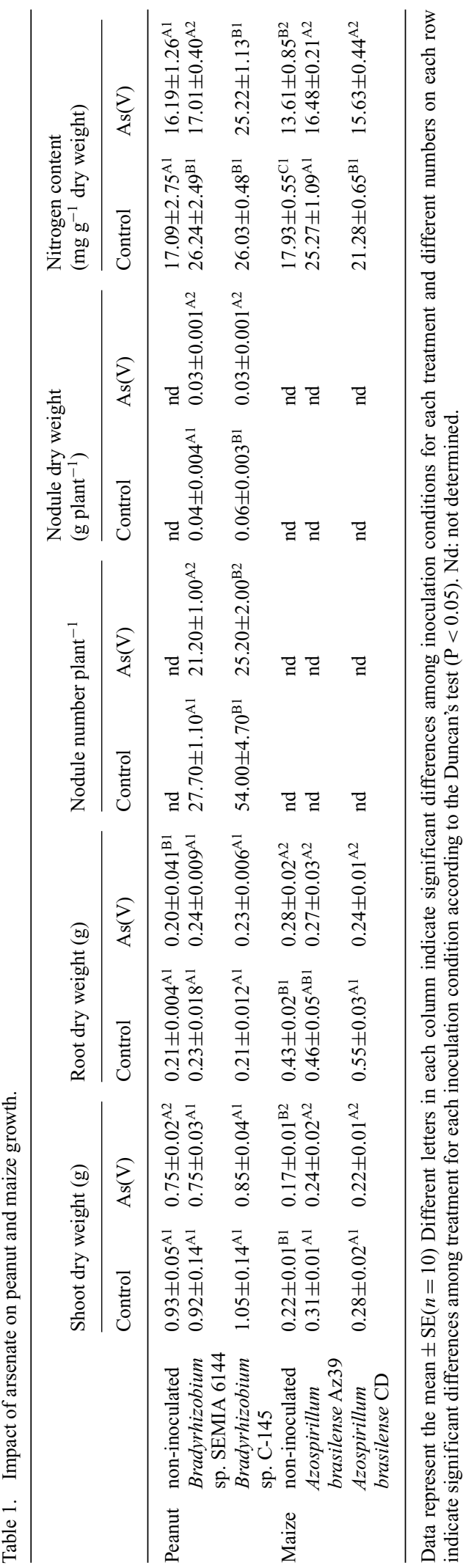


Table 2. Arsenic accumulation and translocation in peanut and maize plants exposed to As(V).

\begin{tabular}{|c|c|c|c|c|c|}
\hline & & \multicolumn{4}{|c|}{ Arsenic accumulation ( $\mu \mathrm{g} \mathrm{g}^{-1}$ dry weight) } \\
\hline & & Shoot & Root & Nodule & TF \\
\hline \multirow[t]{3}{*}{ Peanut } & non-inoculated & $18.64 \pm 0.62^{\mathrm{b}}$ & $64.45 \pm 1.22^{\mathrm{c}}$ & nd & $0.29 \pm 0.007^{\mathrm{a}}$ \\
\hline & Bradyrhizobium sp. SEMIA 6144 & $15.04 \pm 0.39^{\mathrm{b}}$ & $178.85 \pm 4.00^{\mathrm{a}}$ & $130.70 \pm 10.85^{\mathrm{a}}$ & $0.08 \pm 0.002^{\mathrm{b}}$ \\
\hline & Bradyrhizobium sp. C-145 & $34.41 \pm 2.25^{\mathrm{a}}$ & $121.47 \pm 4.34^{\mathrm{b}}$ & $81.70 \pm 1.24^{\mathrm{b}}$ & $0.28 \pm 0.017^{\mathrm{a}}$ \\
\hline \multirow[t]{3}{*}{ Maize } & non-inoculated & $9.14 \pm 0.39^{\mathrm{a}}$ & $7.21 \pm 0.16^{\mathrm{c}}$ & nd & $1.26 \pm 0.046^{\mathrm{a}}$ \\
\hline & Azospirillum brasilense Az39 & $7.52 \pm 0.34^{\mathrm{b}}$ & $14.19 \pm 0.55^{b}$ & nd & $0.53 \pm 0.015^{b}$ \\
\hline & Azospirillum brasilense CD & $10.16 \pm 0.23^{\mathrm{a}}$ & $33.27 \pm 0.83^{\mathrm{a}}$ & nd & $0.30 \pm 0.003^{\mathrm{c}}$ \\
\hline
\end{tabular}

Data represent the mean $\pm S E(n=10)$ Different letters in each column indicate significant differences among inoculation conditions for each tissue according to the Duncan's test $(\mathrm{P}<0,05)$. Nd: not determined.

inoculated with Bradyrhizobium sp. C-145 was higher than that found in other inoculation conditions. On the other hand, roots and nodules of plants inoculated with Bradyrhizobium sp. SEMIA6144 presented the highest As content. Interestingly, inoculated plants revealed higher As content compared to non-inoculated plants in below-ground organs. Regarding As translocation from roots to shoots, inoculation of peanut plants with Bradyrhizobium sp. SEMIA6144 revealed a significant lower TF among inoculation conditions proved (Table 2).

In inoculated maize plants, metalloid accumulation was mainly detected in roots compared with shoots. On the contrary, non-inoculated plants accumulated higher As content on shoot than roots. The comparison between inoculated strains revealed that shoot and root As content of plants inoculated with A. brasilense $\mathrm{CD}$ was higher than that found with A. brasilense Az39. As observed in peanut plants, inoculated maize plants presented higher As content in roots compared to non-inoculated plants. Regarding As translocation from roots to shoots, inoculation of maize plants decreased metalloid $\mathrm{TF}$ in a significant way compared to non-inoculated plants. Metalloid distribution pattern in the studied crops are in agreement with that found in non-hyperaccumulating legumes (Bianucci et al., 2017, 2018; Mandal et al., 2008) and in poaceae (Derlicková et al., 2013). Taking into account the results presented, Bradyrhizobium sp. SEMIA6144 could constitute a promising inoculant for peanut plant in order to decrease As translocation to shoots avoiding fruit contamination. Although, maize inoculation with both tested PGPB avoid metalloid translocation to aerial part of the plant, $A$. brasilense Az39 also promoted growth. Thus, it is possible to suggest that the selection of the best PGPB-plant interaction that not only improve growth but also reduce As accumulation in shoots and therefore in the harvest product, could be a promising biotechnological tool to be used in contaminated field.

\section{CONCLUSIONS}

Arsenic negatively impacts on peanut and maize growth and the inoculation with PGPB represents an effective and promising strategy to improve plant development by restraining metalloid translocation to edible parts. In this sense, the rhizoinoculation with Bradyrhizobium sp. SEMIA6144 in peanut plants and Azospirillum Az39 in maize, results a biotechnological approach to prevent metalloid translocation avoiding As distribution in the trophic chain.

Thus to prepare a strategy for safe tubewell installation leading to the development of a method combining hydrogeological suitability and social mapping which allowed to optimize the locations for safe well installations.

\section{ACKNOWLEDGEMENTS}

This research was supported by Secretaría de Ciencia y Técnica de la Universidad Nacional de Río Cuarto (SECYT-UNRC), Consejo Nacional de Investigaciones Científicas y Técnicas (CONICET) and Fondo para la Investigación Científica y Tecnológica (FONCYT) PICT 2014-0956.

\section{REFERENCES}

Bianucci, E., Furlan, A., Tordable, M.C., Hernández, L.E., Carpena-Ruiz, R.O. \& Castro, S. 2017. Antioxidant responses of peanut roots exposed to realistic groundwater doses of arsenate: identification of glutathione Stransferase as a suitable biomarker for metalloid toxicity. Chemosphere 181: 551-561.

Bianucci, E. Godoy, A., Furlan, A., Peralta, J.M., Hernández, L., Carpena-Ruiz, R.O \& Castro S. 2018. Arsenic toxicity in soybean alleviated by a symbiotic species of Bradyrhizobium. Symbiosis 74(3): 167-176.

Blarasin, M., Cabrera, A. \& Matteoda, E. 2014. Aguas subterráneas de la provincia de Córdoba. UniRío. Universidad Nacional de Río Cuarto. Argentina.

Carpena, R., Esteban, E., Lucena, J.J., Peñalosa, S., Vázquez, P., Zornoza, P. \& Gárate, A. 2006. Simbiosis y fitorrecuperación de suelos. Fijación de Nitrógeno: Fundamentos y Aplicaciones. Sociedad Española de Fijación del Nitrógeno, pp. 255-268.

Döbereiner, J. 1988. Isolation and identification of root associated diazotrophs. Plant Sci. 110: 207-212.

Drličková, G., Vaculík, M., Matejkovic, P. \& Lux, A. 2013. Bioavailability and toxicity of arsenic in maize (Zea mays 
L.) grown in contaminated soils. Bull. Environ. Contam. Toxicol. 91: 235-223.

FAO. 2015. http://faostat.fao.org.

Finnegan, P. \& Chen, W. 2012. Arsenic toxicity: the effects on plant metabolism. Front. Physiol. 3: 182.

Glick, B.R. 1995. The enhancement of plant growth by freeliving bacteria. Can. J. Microbiol. 41(2): 109-117.

Hoagland, D. \& Arnon, D. 1950. The water-culture method for growing plants without soil. California Agricultural Experiment Station.

Lu, H.-D., Xue, J.-Q. \& Guo, D.-W. 2017. Efficacy of planting date adjustment as a cultivation strategy to cope with drought stress and increase rainfed maize yield and water-use efficiency. Agric. Water Manag. 179: 227-235.

Mandal, S.M., Pati, B.R., Das, A.K. \& Ghosh, A.K. 2008. Characterization of a symbiotically effective Rhizobium resistant to arsenic: isolated from the root nodules of Vigna mungo (L.) Hepper grown in an arsenic-contaminated field. J. Gen. Appl. Microbiol. 54(2): 93-99.
Singh, R. \& Agrawal, M. 2007. Effects of sewage sludge amendment on heavy metal accumulation and consequent responses of Beta vulgaris plants. Chemosphere 67(11): 2229-2240.

Sobrino-Plata, J., Ortega-Villasante, C., Flores-Cáceres, M.L., Escobar, C., Del Campo, F.F. \& Hernández, L.E. 2009. Differential alterations of antioxidant defenses as bioindicators of mercury and cadmium toxicity in alfalfa. Chemosphere 77(7): 946-954.

Vásquez-Reina, S., Esteban, E. \& Goldsbrough, P. 2005. Arsenate-induced phytochelatins in white lupin: influence of phosphate status. Physiol. Plant. 124(1): 41-49.

Vincent, J. 1970. A manual for the practical study of the rootnodule bacteria. In: A Manual for the Practical Study of the Root-Nodule Bacteria. Blackwell Scientific Publications Ltd, pp. 73-97. 